\title{
Stereospecific recognition of pyochelin and enantio-pyochelin by the PchR proteins in fluorescent pseudomonads
}

Correspondence

Cornelia Reimmann

Cornelia.Reimmann@unil.ch

Received 30 December 2009

Revised 12 February 2010

Accepted 2 March 2010

\section{Zeb A. Youard and Cornelia Reimmann}

\author{
Département de Microbiologie Fondamentale, Université de Lausanne, CH-1015 Lausanne, \\ Switzerland
}

\begin{abstract}
The siderophore pyochelin of Pseudomonas aeruginosa promotes growth under iron limitation and induces the expression of its biosynthesis genes via the transcriptional AraC/XylS-type regulator PchR. Pseudomonas fluorescens strain CHAO makes the optical antipode of pyochelin termed enantio-pyochelin, which also promotes growth and induces the expression of its biosynthesis genes when iron is scarce. Growth promotion and signalling by pyochelin and enantio-pyochelin are highly stereospecific and are known to involve the pyochelin and enantiopyochelin outer-membrane receptors FptA and FetA, respectively. Here we show that stereospecificity in signalling is also based on the stereospecificity of the homologous PchR proteins of $P$. aeruginosa and $P$. fluorescens towards their respective siderophore effectors. We found that PchR functioned in the heterologous species only if supplied with its native ligand and that the FptA and FetA receptors enhanced the efficiency of signalling. By constructing and expressing hybrid and truncated PchR regulators we showed that the weakly conserved Nterminal domain of PchR is responsible for siderophore specificity. Thus, both uptake and transcriptional regulation confer stereospecificity to pyochelin and enantio-pyochelin biosynthesis.
\end{abstract}

\section{INTRODUCTION}

Iron is essential for the growth of most living organisms, including bacteria. Although an abundant element in nature, its bioavailability for bacteria growing in aerobic conditions is low due to poor solubility (about $1.4 \times 10^{-9}$ $\mathrm{M}$ at pH 7.0 for ferric iron; Chipperfield \& Ratledge, 2000). For pathogenic bacteria iron can be even scarcer due to it being bound to iron storage and transport proteins within mammalian hosts. To acquire sufficient iron for growth, bacteria have evolved a number of strategies, the most common of which is the production of low-molecularmass iron-chelating molecules termed siderophores. Under iron limitation siderophores are secreted by the bacterium into the extracellular environment, where they chelate ferric iron and return it to the cell via specific receptor proteins in the cell membrane.

Pyochelin, 2-(2-o-hydroxyphenyl-2-thiazolin-4-yl)-3methylthiazolidine-4-carboxylic acid (Cox et al., 1981), is a thiazoline siderophore produced by the opportunistic pathogen Pseudomonas aeruginosa PAO1 (ATCC 15692) and other closely related species (Sokol, 1986; Castignetti, 1997; Darling et al., 1998). Pyochelin chelates ferric iron in solution with a 2:1 siderophore: ligand stoichiometry (Schlegel et al., 2006; Tseng et al., 2006) and a dissociation constant of $2.4 \times 10^{5} \mathrm{M}^{-1}$

A supplementary table of primers and a supplementary figure are available with the online version of this paper. in ethanol (Cox \& Graham, 1979). This is low compared to the iron affinity of pyoverdine, the primary siderophore of $P$. aeruginosa (dissociation constant $\sim 10^{24} \mathrm{M}^{-1}$ at $\mathrm{pH}$ 7.0; Visca et al., 2007), but the iron affinity of pyochelin in aqueous solution at physiological $\mathrm{pH}$ is unknown and may be higher. Pyochelin is a condensation product of salicylate and two cysteinyl residues and its biosynthesis in $P$. aeruginosa requires proteins encoded by the operons pchDCBA and pchEFGHI (Serino et al., 1995, 1997; Reimmann et al., 1998, 2001). Pyochelin contains three chiral centres $\left(\mathrm{C}^{\prime}, \mathrm{C} 2^{\prime \prime}\right.$ and $\left.\mathrm{C}^{\prime \prime}\right)$ and exists in solution as a mixture of two interconvertible stereoisomers (Fig. 1): pyochelin I $\left(4^{\prime} R, 2^{\prime \prime} R, 4^{\prime \prime} R\right)$ and pyochelin II $\left(4^{\prime} R, 2^{\prime \prime} S, 4^{\prime \prime} R\right)$ (Ankenbauer et al., 1988; Rinehart et al., 1995) although only pyochelin I has been observed to bind ferric iron (Schlegel et al., 2004, 2006).

Upon iron chelation, pyochelin is transported back into the cell via a specific outer-membrane receptor, FptA, in conjunction with an inner-membrane permease, FptX (Ankenbauer \& Quan, 1994; Ó Cuiv et al., 2004; Michel et al., 2007). Pyochelin then upregulates its own biosynthesis and uptake genes by activating the AraC/XylS-family transcriptional regulator PchR. Activated PchR binds to a conserved sequence motif (PchR-box) in the promoter regions of $p c h D C B A$, pchEFGHI, $f p t A B C X$ and $p c h R$ and regulates transcription of these genes (Michel et al., 2005, 2007). Unlike AraC, the prototype of this family (Schleif, 2003), PchR appears to have only one binding site in these 
<smiles>CN1C(C(=O)O)(C(=O)O)CSC1(C(C)(Cl)Cl)C1(C(Cl)(Cl)Cl)CSC(c2ccccc2O)=N1</smiles><smiles>CN1[C@H](C2CSC(c3ccccc3O)=N2)SC[C@H]1C(=O)O</smiles>

Pyochelin II (4'R, 2"S, 4"R)<smiles>CN1[C@@H](C(=O)O)CS[C@H]1[C@H]1CSC(c2ccccc2O)=N1</smiles>

Enantio-pyochelin II $\left(4^{\prime} S, 2^{\prime \prime} R, 4^{\prime \prime} S\right)$<smiles>CN1[C@H](C(=O)O)CS[C@@H]1[C@H]1CSC(c2ccccc2O)=N1</smiles>

Pyochelin I (4'R, 2"R, 4"R)<smiles>CN1C(C(=O)O)SC[C@H]1[C@H]1CSC(c2ccccc2O)=N1</smiles>

Enantio-pyochelin I (4'S, 2"S, 4"S)

Fig. 1. Structures and configurations of pyochelin and enantio-pyochelin. Pyochelin and enantio-pyochelin each contain three chiral carbons $\left(\mathrm{C4}^{\prime}, \mathrm{C}^{\prime \prime}\right.$ and $\left.\mathrm{C}^{\prime \prime}\right)$ and exist as a pair of spontaneously interconvertible stereoisomers. Epimerization upon binding of metal $\left(\mathrm{M}^{\mathrm{n}+}\right)$ has only been shown for pyochelin (Schlegel et al., 2004, 2006) but may also occur with enantiopyochelin.

promoters and there is no evidence so far for DNA looping. Moreover, it is currently unknown whether PchR, like AraC, is functional as a dimer.

Recently, we reported that the plant-beneficial bacterium $P$. fluorescens CHA0 produces the optical antipode of pyochelin termed enantio-pyochelin (Fig. 1; Youard et al., 2007). Enantio-pyochelin was found to promote growth of $P$. fluorescens under iron limitation and to upregulate its own biosynthesis genes in a manner similar to that of pyochelin in P. aeruginosa (Youard et al., 2007). However, enantio-pyochelin does not promote growth of $P$. aeruginosa and pyochelin has no such activity in $P$. fluorescens (Youard et al., 2007). This stereospecificity correlates with the structural specificity of the pyochelin and enantio-pyochelin receptors. The pyochelin receptor FptA does not bind and transport iron-loaded enantiopyochelin in $P$. aeruginosa, whereas the enantio-pyochelin receptor FetA does not bind and transport ferri-pyochelin in $P$. fluorescens. Swapping the pyochelin and the enantiopyochelin uptake systems between the two bacterial species inverted their siderophore preference and allowed $P$. aeruginosa to utilize enantio-pyochelin as an iron source while $P$. fluorescens was enabled to grow with iron-loaded pyochelin (Hoegy et al., 2009).

Stereospecificity is also observed in signalling with pyochelin and enantio-pyochelin. Addition of enantio- pyochelin to $P$. aeruginosa cultures does not induce the expression of the pyochelin biosynthesis genes and addition of pyochelin to $P$. fluorescens cultures hardly induces the expression of the enantio-pyochelin biosynthesis genes (Youard et al., 2007). It has not been clear, however, if this stereospecificity in signalling might be caused simply by the failure of FptA and FetA to internalize the siderophore of the other species, thereby preventing the activation of the PchR regulatory protein, or whether additionally the PchR protein might not be activated by the siderophore of the other species.

Here we show that stereospecificity in signalling is based, in addition, on the specificity of the PchR proteins of $P$. aeruginosa and $P$. fluorescens towards their siderophore ligand and we investigate how these proteins can distinguish between the two enantiomers. By constructing hybrid and truncated PchR proteins we obtained evidence for the N-terminal part of PchR being responsible for siderophore recognition.

\section{METHODS}

Bacterial strains, plasmids and culture conditions. Bacterial strains and plasmids used in this study are listed in Table 1. Bacteria were grown on nutrient agar and in nutrient yeast broth at $37{ }^{\circ} \mathrm{C}(P$. aeruginosa and Escherichia coli) or $30{ }^{\circ} \mathrm{C}$ (P. fluorescens) (Stanisich \& Holloway, 1972). For green fluorescent protein (GFP) reporter assays, 
strains were cultivated in the complex medium GGP (Carmi et al., 1994), in which iron is present but not immediately accessible, thus inducing the expression of siderophore biosynthesis genes. Antibiotics, when required, were added to the growth media at the following concentrations: ampicillin (Ap) $100 \mu \mathrm{g} \mathrm{ml} \mathrm{m}^{-1}$, gentamicin (Gm) $10 \mu \mathrm{g} \mathrm{ml}^{-1}$ and tetracycline (Tc) $25 \mu \mathrm{g} \mathrm{ml}^{-1}$ for E. coli; Tc $125 \mu \mathrm{g} \mathrm{ml}^{-1}$ and $\mathrm{Gm} 10 \mu \mathrm{g} \mathrm{ml}^{-1}$ for P. aeruginosa and P. fluorescens; kanamycin $(\mathrm{Km}) 50 \mu \mathrm{g} \mathrm{ml}^{-1}$ for $P$. fluorescens. To counterselect $E$. coli donor cells in gene replacement experiments, chloramphenicol $(\mathrm{Cm})$ was used at a concentration of $10 \mu \mathrm{g} \mathrm{ml}^{-1}$; mutant enrichment was performed with Tc at a final concentration of $20 \mu \mathrm{g} \mathrm{ml}^{-1}$ and carbenicillin (for P. aeruginosa) or cycloserine (for P. fluorescens) at final concentrations of $2 \mathrm{mg} \mathrm{ml}^{-1}$ and $50 \mathrm{mg} \mathrm{ml}^{-1}$, respectively. Pyochelin and enantio-pyochelin were purified from culture supernatants of $P$. aeruginosa and $P$. fluorescens, respectively, as described previously (Youard et al., 2007) and added to the growth medium at a final concentration of $20 \mu \mathrm{M}$. This concentration, which is at least 20fold below the physiological concentration reached under iron starvation (Reimmann et al., 1998 and our unpublished results), fully induces the expression of pyochelin and enantio-pyochelin biosynthesis genes in $P$. aeruginosa and $P$. fluorescens, respectively (Youard et al., 2007) and restores growth under iron limitation in siderophore-negative mutants (Hoegy et al., 2009). For IPTG-induced expression of proteins in GGP medium a concentration of $1 \mathrm{mM}$ was used; for IPTG-induced expression of proteins on Chrome Azurol S (CAS) agar (Schwyn \& Neilands, 1987), each plate was surface-spread with $100 \mu \mathrm{l}$ of a $500 \mathrm{mM}$ IPTG stock solution.

DNA manipulations and sequencing. Oligonucleotides used in this study are listed in Supplementary Table S1, available with the online version of this paper. Small- and large-scale preparations of plasmid DNA were made with the QIAprep Spin Miniprep kit (Qiagen) and Jetstar kit (Genomed), respectively. DNA fragments were purified from agarose gels using the MinElute and QIAquick Gel Extraction kits from Qiagen. DNA manipulations were performed according to standard procedures (Sambrook et al., 2001). Transformation of E. coli, $P$. aeruginosa and P. fluorescens was carried out by electroporation (Farinha \& Kropinski, 1990). All constructs involving PCR techniques were verified by sequence analysis. Sequencing was performed with the BigDye Terminator Cycle Sequencing kit and an ABI-PRISM 373 automatic sequencer (Applied Biosystems) or was carried out commercially by Microsynth AG. Sequence alignments were performed using the program BLAST 2 Sequences (Tatusova \& Madden, 1999) available at the National Center for Biotechnology Information (NCBI, http://www.ncbi.nlm.nih.gov).

Mutant constructions. Gene replacement mutants of $P$. aeruginosa and $P$. fluorescens were generated with suicide plasmids as described previously (Schnider et al., 1995; Ye et al., 1995; Laville et al., 1998). All gene replacement mutants were checked by PCR. The suicide plasmid pME9253, used to generate an 897 bp deletion in the pchR gene of $P$. fluorescens, was constructed as follows. Two PCR fragments were generated from chromosomal DNA of $P$. fluorescens CHA0 using the primer pairs pchR1/pchR2 and pchR3/pchR4. The two fragments were then combined by overlap extension PCR using an overhang on the first fragment. The combined fragment, cleaved with HindIII and BamHI, was cloned into the suicide vector pME3087 between the HindIII and BamHI sites. Plasmid pME9253 was then mobilized from E. coli $\mathrm{DH} 5 \alpha$ into the P. fluorescens strains CHA400 and CHA1084 using the helper plasmid pME497 and chromosomally integrated with selection for tetracycline resistance. Excision of the vector via a second crossing-over was obtained by enrichment for tetracycline-sensitive cells to generate the mutants CHA1222 ( $\Delta p c h R, p v d:: \operatorname{Tn} 1733)$ and CHA1223 ( $\triangle$ pchR, $\triangle$ pchDHIEFKCBA), respectively. The previously described suicide plasmids pME7531 (Hoegy et al., 2009) and pME7152 (Michel et al., 2007) were used in a similar way to delete the enantio-pyochelin receptor gene in P. fluorescens CHA0 and the pyoverdine biosynthesis gene $p v d F$ in $P$. aeruginosa PAO6274, generating the corresponding mutants CHA1086 ( $\Delta$ fetA) and PAO6402 (pchR:: $\omega, \Delta p v d F)$, respectively.

Cloning of pch $\boldsymbol{R}_{\text {CHAO. The }} p c h R$ gene of $P$. fluorescens CHA0 and its complete upstream intergenic region were amplified from genomic DNA of P. fluorescens CHA0 by PCR using the primers pchR5 and pchR6. This fragment was cleaved with KpnI and HindIII and cloned into the KpnI- and HindIII-cut chromosomal integration vector pME3280a to generate plasmid pME9252.

Swapping the promoter and leader regions between $p c h R_{\text {PAO1 }}$ and $\boldsymbol{p} \boldsymbol{c h} \boldsymbol{R}_{\text {CHAO. }}$ Plasmid pME9254 containing pchR of P. aeruginosa fused to the upstream intergenic region (promoter and leader) of $P$. fluorescens pchR was constructed as follows. Two PCR fragments were generated from chromosomal DNA of $P$. fluorescens CHA0 and P. aeruginosa PAO1, respectively. Fragment 1 was amplified using the primers pchR7 and pchR8 and fragment 2 with primers pchR9 and pchR10. The two fragments were then combined by overlap extension PCR using an overhang on the first fragment and cleaved with HindIII and KpnI. Finally, the cleaved fragment was inserted into pME3280a between the HindIII and KpnI sites. Plasmid pME9255 containing $p c h R$ of $P$. fluorescens fused to the upstream intergenic region of $P$. aeruginosa $p c h R$ was constructed in a similar way. PCR fragment 1 was amplified from $P$. aeruginosa using the primers pchR11 and pchR12 and fragment 2 was amplified from $P$. fluorescens with primers pchR13 and pchR14. The two fragments were combined by overlap extension PCR using an overhang on the first fragment, cleaved with HindIII and KpnI and ligated into pME3280a.

Construction of $\mathbf{N}$-terminal PchR expression plasmids. To construct plasmid pME9264, nucleotides 1-609 of $p c h R_{\mathrm{PAO} 1}$ were amplified by PCR from chromosomal DNA of $P$. aeruginosa PAO1 using the primers pchR36a and pchR37. This fragment was cleaved with EcoRI and BamHI and ligated into EcoRI- and BamHI-cut pME6032. The resulting plasmid expresses amino acids 1-203 of $\mathrm{PchR}_{\mathrm{PAO} 1}$ fused to a C-terminal $\mathrm{His}_{6}$ tag provided by primer pchR37. Plasmid pME9265 expressing amino acids 1-207 of PchR $\mathrm{CHA0}_{\text {fused }}$ to a C-terminal $\mathrm{His}_{6}$ tag was obtained in a similar way. Nucleotides 1621 of $p c h R_{\text {CHAo }}$ were PCR-amplified from $P$. fluorescens CHA0 using the primers pchR38a and pchR39. The resulting DNA fragment was then trimmed with EcoRI and BamHI and cloned into pME6032.

Construction of hybrid pchR genes. Hybrid $p c h R$ genes were constructed by overlap PCR. DNA from $p c h R_{\mathrm{PAO} 1}$ and $p c h R_{\mathrm{CHA} 0}$ was fused at locations (determined by sequence alignment) that encoded identical amino acid sequences in both PchR proteins (see Fig. 5). For hybrid $p c h R s$ 1-3 two DNA fragments were amplified, one containing the upstream intergenic region of $p c h R_{\mathrm{CHAO}}$ and a $5^{\prime}$ section of the adjacent $p c h R$ gene (amplified from $P$. fluorescens CHA0 genomic DNA), and a second encoding a $3^{\prime}$ section of $p c h R_{\mathrm{PAO}}$ (amplified from $P$. aeruginosa $\mathrm{PAO} 1$ genomic DNA) including the stop codon. The second fragment was amplified using a forward primer with a $5^{\prime}$ overlap sequence identical to the $3^{\prime}$ end of the first PCR fragment. The two PCR products were then combined with a second PCR cycle, cleaved with HindIII and SpeI and ligated into HindIII- and SpeI-cut pME3280a. Hybrid 1 (pME9263) was generated using primers pchR15, pchR34, pchR35 and pchR25, hybrid 2 (pME9258) using primers pchR15, pchR21, pchR22 and pchR25, and hybrid 3 (pME9260) using primers pchR15, pchR40, pchR41 and pchR25.

For hybrids 1a-3a the method was the same as described above except that pME9254 was used as the template (upstream intergenic region of $p c h R_{\mathrm{CHA} 0}$ fused to $\left.p c h R_{\mathrm{PAO} 1}\right)$ for the first fragment and chromosomal DNA of $P$. fluorescens $\mathrm{CHA} 0$ was used as the template for the second. Hybrid la (pME9266) was constructed using primers pchR42, pchR54, pchR55 and pchR45, hybrid 2a (pME9269) using 
Table 1. Bacterial strains and plasmids

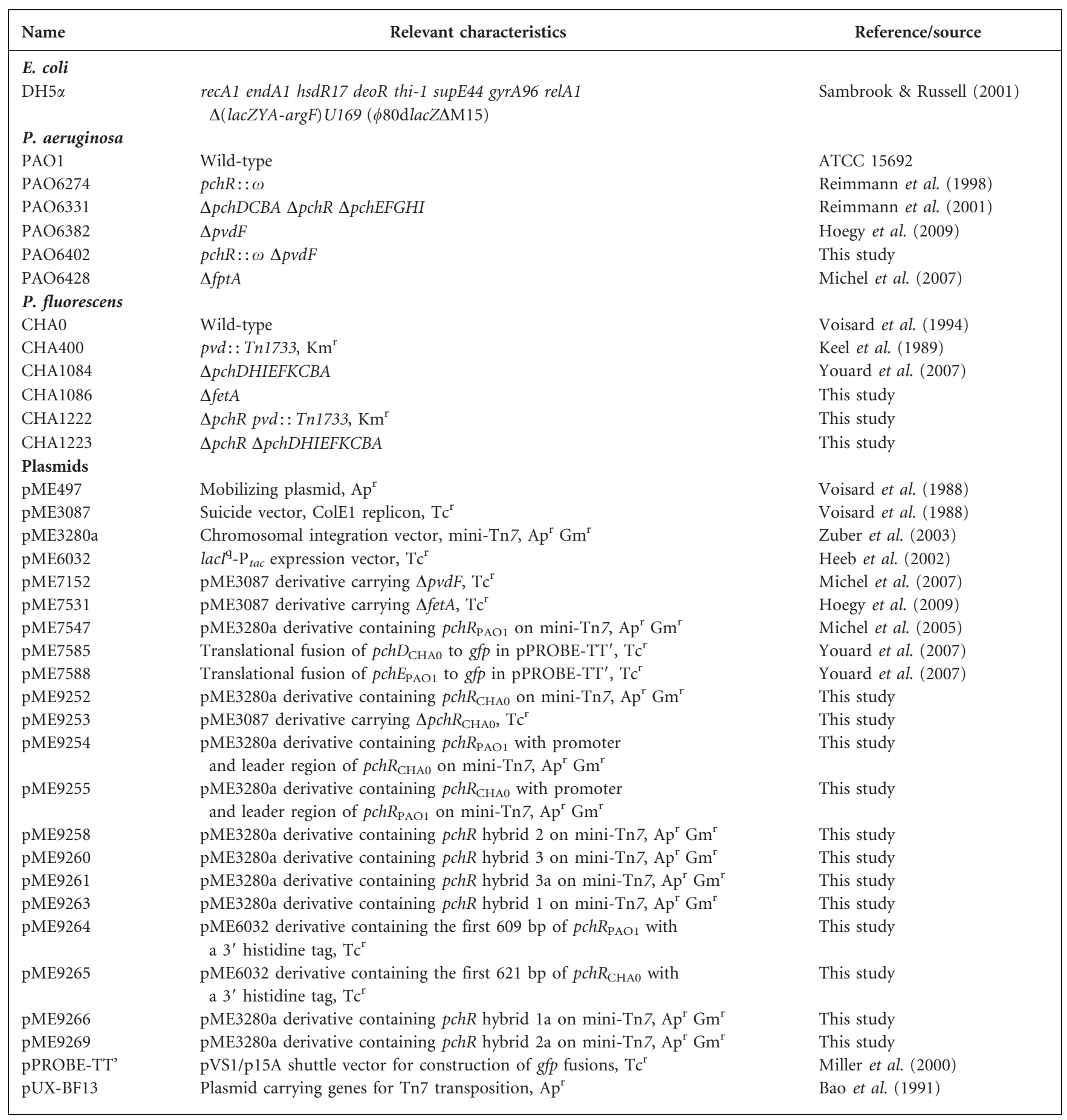

primers pchR42, pchR48, pchR49 and pchR45, and hybrid 3a (pME9261) using primers pchR42, pchR43, pchR44 and pchR45.

Wild-type and hybrid $p c h R$ genes were integrated as mini-Tn7derivatives into the chromosome of $P$. aeruginosa and $P$. fluorescens as described previously, using plasmid pUX-BF13 containing the genes for $\operatorname{Tn} 7$ transposition (Zuber et al., 2003).

GFP reporter assays. Pseudomonas strains carrying translational $g f p$ fusions were grown in 96-well black microtitre plates (Greiner bio-one) with a flat transparent bottom. Each well contained $200 \mu$ l GGP medium and was inoculated with $3 \mu \mathrm{l}$ of a bacterial culture grown overnight in the same medium. HPLC-purified pyochelin or enantiopyochelin was dissolved in methanol to a concentration of $10 \mathrm{mM}$ (determined by the CAS assay; Schwyn \& Neilands, 1987) and added to the growth medium at the concentrations indicated. Microtitre plates were incubated at $37{ }^{\circ} \mathrm{C}\left(P\right.$. aeruginosa) or $30{ }^{\circ} \mathrm{C}$ (P. fluorescens) with orbital shaking at 500 r.p.m. At each given time point, growth $\left(\mathrm{OD}_{600}\right)$ and green fluorescence (excitation at $480 \mathrm{~nm}$ and emission at $520 \mathrm{~nm}$ ) were measured from triplicate cultures using a Fluostar fluorescence microplate reader (BMG Lab Technologies). For each individual measurement the green fluorescence value was divided by the respective 


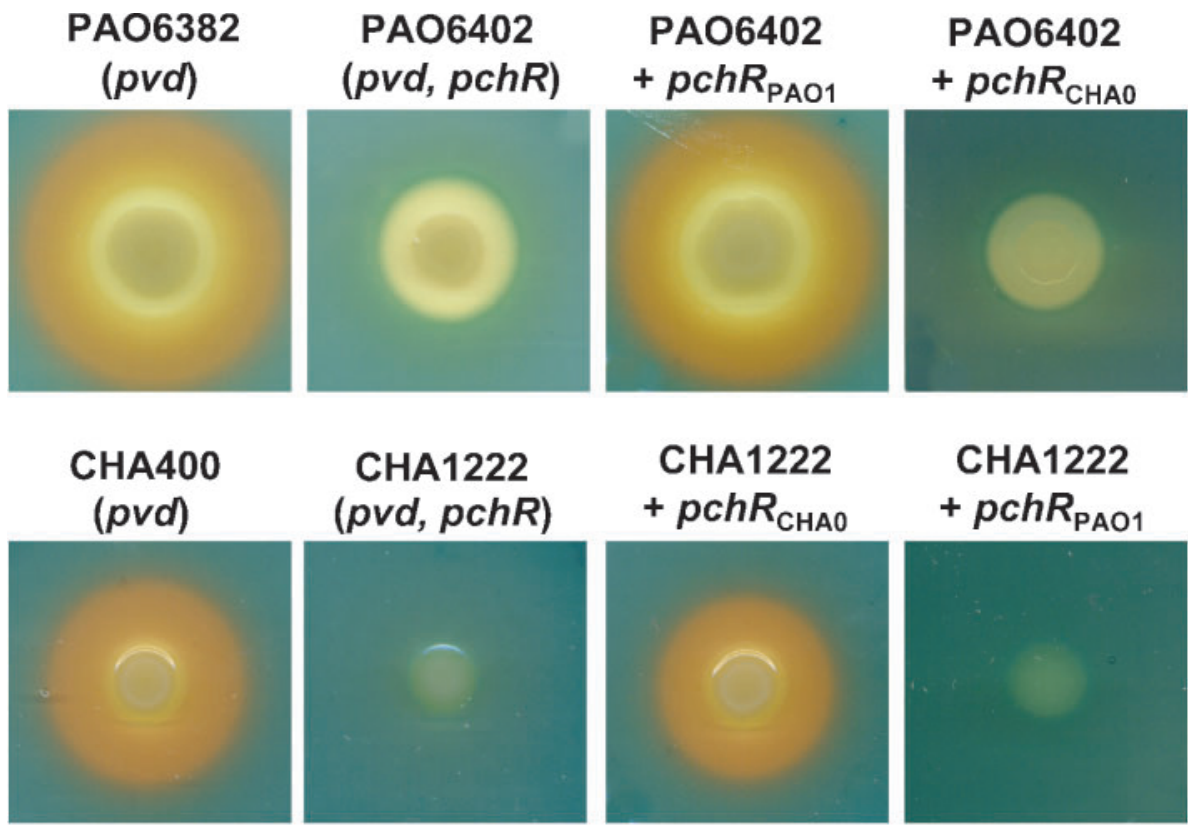

Fig. 2. Siderophore production of $P$. aeruginosa and $P$. fluorescens $p c h R$ mutants complemented by homologous and heterologous pchR genes. The pchR genes of $P$. aeruginosa and $P$. fluorescens carried by pME7547 and pME9252, respectively, were tested for their ability to complement the pyoverdine-negative (pvd) pchR mutants PAO6402 and CHA1222. Pyochelin and enantio-pyochelin production can be visualized by the formation of an orange halo surrounding the colony. The pyoverdine-negative parent strains PAO6382 and CHA400 served as positive controls. The experiment was repeated twice with very similar results. Note that pyoverdine-negative strains were used to ensure that the haloes observed on CAS agar were caused by pyochelin or enantio-pyochelin.

$\mathrm{OD}_{600}$ value to give the specific fluorescence of cells expressed as relative fluorescence units. The green fluorescence of cells carrying the empty vector pPROBE-TT' was determined for background fluorescence correction.

Test for siderophore production on CAS agar. CAS agar plates were prepared as described by Schwyn \& Neilands (1987). CAS agar contains a blue-coloured iron dye complex (chrome azurol Siron(III)-hexadecyltrimethylammonium bromide) that changes colour to orange upon formation of an iron-ligand complex and release of the dye. Strains were tested for siderophore production by dotting $2 \mu \mathrm{l}$ of an overnight culture (grown in iron-limited GGP medium) onto CAS agar and incubating the plate overnight at the appropriate temperature. Siderophore-producing strains were identified by the presence of an orange halo surrounding the colony.

\section{RESULTS}

\section{The pchR genes of $P$. aeruginosa and $P$. fluorescens do not complement pchR mutations in the other species}

To see whether the PchR proteins are specific for pyochelin and enantio-pyochelin, respectively, we first tested if $p c h R$ of $P$. aeruginosa could complement a pchR mutation in $P$. fluorescens and vice versa (Fig. 2). As expected, no siderophore production was detected on CAS agar medium with the pyoverdine-negative $p c h R$ mutant PAO6402, confirming that $p c h R$ is essential for pyochelin production in P. aeruginosa (Heinrichs \& Poole, 1993). Pyochelin production in PAO6402 was entirely restored with $p c h R$ of $P$. aeruginosa $\left(p c h R_{\mathrm{PAO} 1}\right)$ carried by pME7547 and delivered in monocopy to the chromosome of the pchR mutant as a mini-Tn7 derivative (see Methods). By contrast, when $p c h R$ of $P$. fluorescens $\left(p c h R_{\mathrm{CHAO}}\right.$ ) carried by pME9252 was integrated into the chromosome of PAO6402, no complementation occurred, indicating that $\mathrm{PchR}_{\mathrm{CHA} 0}$ does not function in $P$. aeruginosa. Likewise, $p c h R_{\mathrm{PAO} 1}$ was unable to complement the $p c h R$ mutation in the pyoverdine-negative P. fluorescens mutant CHA1222, whereas enantio-pyochelin production was restored in this mutant by $p c h R_{\text {CHAO. }}$ We conclude from these experiments that both $p c h R$ genes are species-specific.

Species-specificity of PchR could be caused (i) by $p c h R$ expression problems in the heterologous host, (ii) by sequence differences between the PchR-boxes of $P$. aeruginosa and $P$. fluorescens, or (iii) by a specificity of PchR for the native ligand. However, the PchR-box sequences of $P$. aeruginosa and $P$. fluorescens are almost identical (Michel et al., 2005) and the $p c h R$ genes were expressed in the heterologous background (see below, Fig. 3). Therefore, it seemed likely that the PchR proteins were specific for their native ligand. 

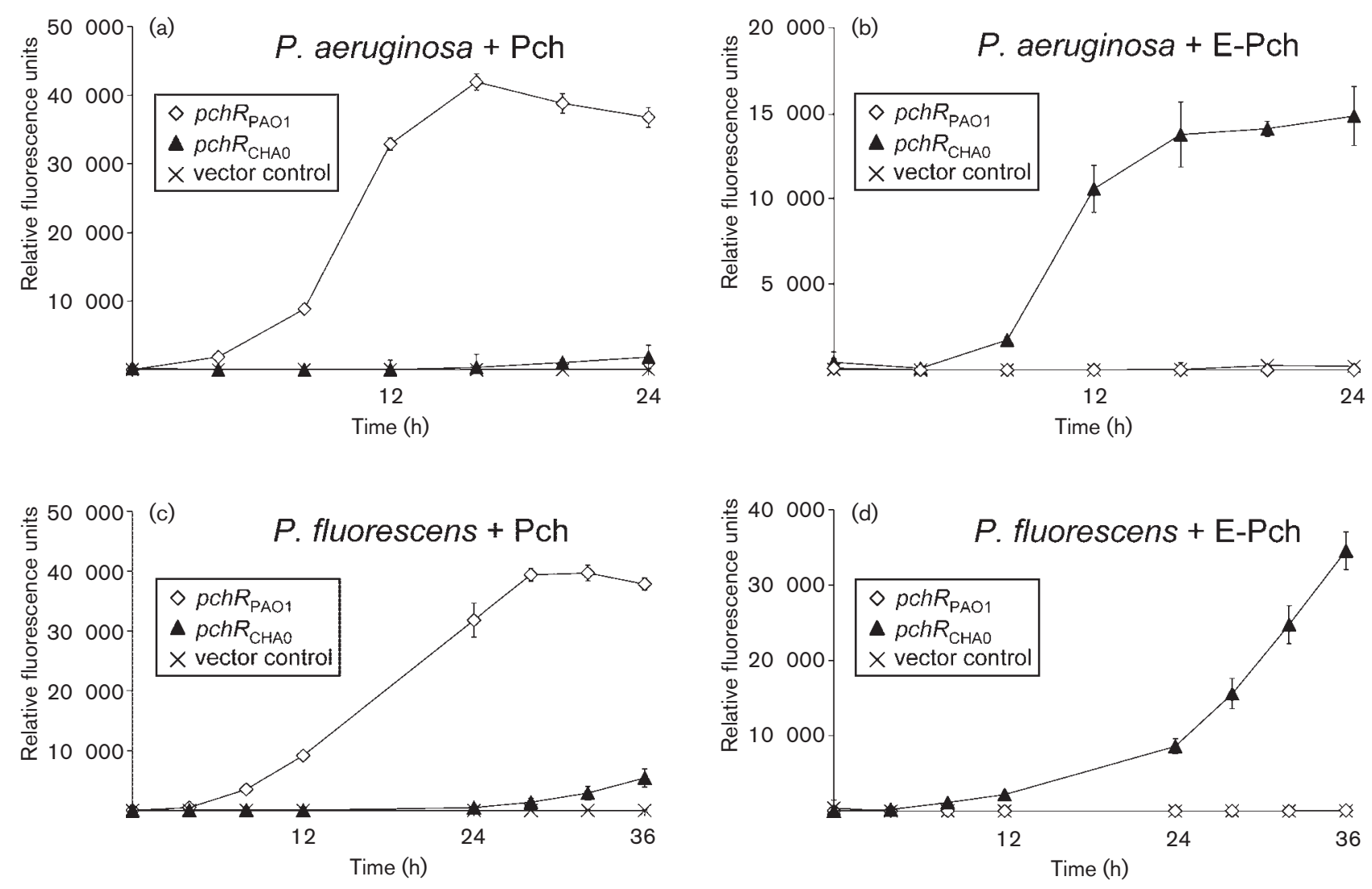

Fig. 3. Specificity of the $P$. aeruginosa and $P$. fluorescens PchR proteins for their native ligand. The pyochelin-negative $p c h R$ mutant of $P$. aeruginosa (PAO6331; a, b) and the enantio-pyochelin-negative pchR mutant of $P$. fluorescens (CHA1223; c, d)

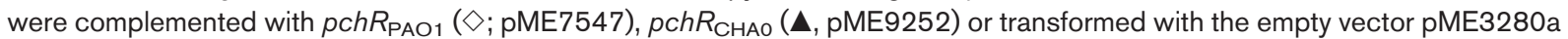
$(\mathbf{x})$ and grown in GGP medium containing $20 \mu \mathrm{M}$ HPLC-purified pyochelin (Pch) (a, c) or enantio-pyochelin (E-Pch) (b, d). $P$. aeruginosa strains contained the reporter plasmid pME7588 with a $p c h E_{\mathrm{PAO} 1}{ }^{\prime}{ }^{\prime} g f p$ translational fusion, $P$. fluorescens strains contained pME7585 with a $p c h D_{\mathrm{CHAO}}{ }^{-}$'gfp translational fusion. Background fluorescence was assessed in both species with the empty vector pPROBE-TT'. Strains were grown in microtitre wells for $24 \mathrm{~h}$, and green fluorescence and growth $\left(\mathrm{OD}_{600}\right)$ were measured from three parallel experiments. Fluorescence is expressed as relative fluorescence units \pm standard deviation.

\section{Specificity of PchR for pyochelin or enantio- pyochelin}

We compared the ability of pyochelin and enantiopyochelin to activate $\mathrm{PchR}_{\mathrm{PAO}}$ and $\mathrm{PchR}_{\mathrm{CHAO}}$ in both Pseudomonas species. Experiments were carried out with the pchR mutant strains PAO6331 and CHA1223, which were grown in iron-limited GGP medium supplemented with HPLC-purified pyochelin or enantio-pyochelin. These strains are also pyochelin- and enantio-pyochelin-negative, respectively, to ensure that the only siderophore able to interact with PchR would be the one supplied to the growth medium. In agreement with previous findings (Michel et al., 2005), monocopy insertion of $p c h R_{\mathrm{PAO} 1}$ into the chromosome of PAO6331 enabled expression of the pyochelin biosynthesis gene $p c h E_{\mathrm{PAO} 1}$ when the strain was grown in medium supplemented with pyochelin (Fig. 3a). Addition of enantio-pyochelin to the growth medium did not promote $p c h E_{\mathrm{PAO} 1}$ expression (Fig. 3b). Conversely, $p c h R_{\mathrm{CHA} 0}$ promoted $p c h E_{\mathrm{PAO} 1}$ expression in PAO6331 when grown with enantio-pyochelin (Fig. 3b) while pyochelin had only a very small effect (Fig. 3a). Stereospecificity of the two PchR proteins was confirmed by experiments carried out in $P$. fluorescens using the enantio-pyochelin biosynthesis gene $p c h D_{\mathrm{CHA} 0}$ as a reporter. As shown in Fig. 3(c, d) pch $R_{\mathrm{PAO}}$ was able to promote the expression of $p c h D_{\mathrm{CHAO}}$ only in the presence of pyochelin, whereas activation of $\mathrm{PchR}_{\mathrm{CHAO}}$ occurred with enantio-pyochelin. Again, $\mathrm{PchR}_{\mathrm{CHA} 0}$ was slightly activated by pyochelin as well, thus confirming our previous findings, which suggested that $\mathrm{PchR}_{\mathrm{CHAO}}$ could have a somewhat more relaxed specificity for its ligand than PchR $\mathrm{PAO}_{\mathrm{P}}$ (Youard et al., 2007).

We conclude from these data that (i) both $p c h R$ genes are expressed in $P$. aeruginosa and $P$. fluorescens, (ii) both PchR proteins are able to recognize the PchR-box in the promoter regions of $p c h E_{\mathrm{PAO} 1}$ and $p c h D_{\mathrm{CHA} 0}$, and (iii) $\mathrm{PchR}_{\mathrm{PAO}}$ is specific for pyochelin while $\mathrm{PchR}_{\mathrm{CHA} 0}$ prefers enantio-pyochelin. Note that expression levels and kinetics 
of the reporter were not identical in the two species, especially not for enantio-pyochelin (Fig. 3b, d), probably because of differences in growth conditions $\left(37^{\circ} \mathrm{C}\right.$ for $P$. aeruginosa vs $30{ }^{\circ} \mathrm{C}$ for $P$. fluorescens), siderophore uptake (receptor-dependent and receptor-independent, see below), PchR-box recognition (different reporter genes were used in the two species), or pchR expression levels.

\section{Stereospecificity of the receptor proteins FptA and FetA is not a prerequisite for stereospecificity in signalling}

The pyochelin and enantio-pyochelin receptors FptA and FetA are specific for binding and transport of their respective ferri-siderophores (Ankenbauer \& Quan, 1994; Hoegy et al., 2009), implying that for interaction with PchR (Fig. 3) both siderophores must also be able to enter the cells via another less specific mechanism. This was confirmed by comparing the expression of the pyochelin and enantio-pyochelin biosynthesis genes in the receptor mutants PAO6428 $(\triangle f p t A)$ and CHA1086 $(\Delta f e t A)$ to that in the wild-type strains PAO1 and CHA0 (Fig. 4). In both mutants, expression of the siderophore biosynthesis genes was delayed, but not abolished, compared to the wild-type strains and addition of pyochelin and enantio-pyochelin, respectively, to the growth medium increased expression in the wild-type and in the receptor mutants (Fig. 4). Moreover, deletion of the receptor genes in pyochelinand enantio-pyochelin-negative mutants, respectively, did not abolish induction of $p c h E_{\mathrm{PAO} 1}$ by pyochelin in $P$. aeruginosa and of $p c h D_{\mathrm{CHA} 0}$ by enantio-pyochelin in $P$. fluorescens, but reduced the dose-response compared to their corresponding receptor-positive parent (data not shown). Thus, the pyochelin/enantio-pyochelin receptors FptA/FetA increase signalling efficiency but they are not essential for signalling because sufficient amounts of both siderophores can bypass the specific receptors and enter the cell through a secondary, as yet unknown mechanism.

\section{Siderophore specificity of PchR is conferred by the $\mathbf{N}$-terminal part of the protein}

Alignment of the deduced amino acid sequences of $P c h R_{\mathrm{PAO} 1}$ and $\mathrm{Pch} \mathrm{R}_{\mathrm{CHAO}}$ showed that the primary sequences of the two regulators are very similar, with most variation occurring in the N-terminal $\sim 20$ residues and highest similarity in the C-terminal $\sim 100$ residues (Fig. $5 \mathrm{a}$ ). On the basis of homology to other members of the AraC/XylS family of proteins (Gallegos et al., 1997) the C-terminal region of PchR is presumed to form the DNA-binding domain, which could allow binding of PchR to the PchRbox in the promoter of pyochelin- and enantio-pyochelinregulated genes. The N-terminal two-thirds of PchR are expected to form the siderophore recognition domain and may also be involved in multimerization, as shown for other members of the AraC/XylS family (Ruíz et al., 2003 and references therein).

To investigate if the PchR N-terminal region is responsible for siderophore binding and stereospecificity we constructed a series of $p c h R$ hybrids encoding PchR proteins with different parts of $\mathrm{PchR}_{\mathrm{PAO} 1}$ and $\mathrm{PchR}_{\mathrm{CHAO}}$. Using the alignment of $\mathrm{PchR}_{\mathrm{PAO} 1}$ and $\mathrm{PchR}_{\mathrm{CHAO}}$ we selected three
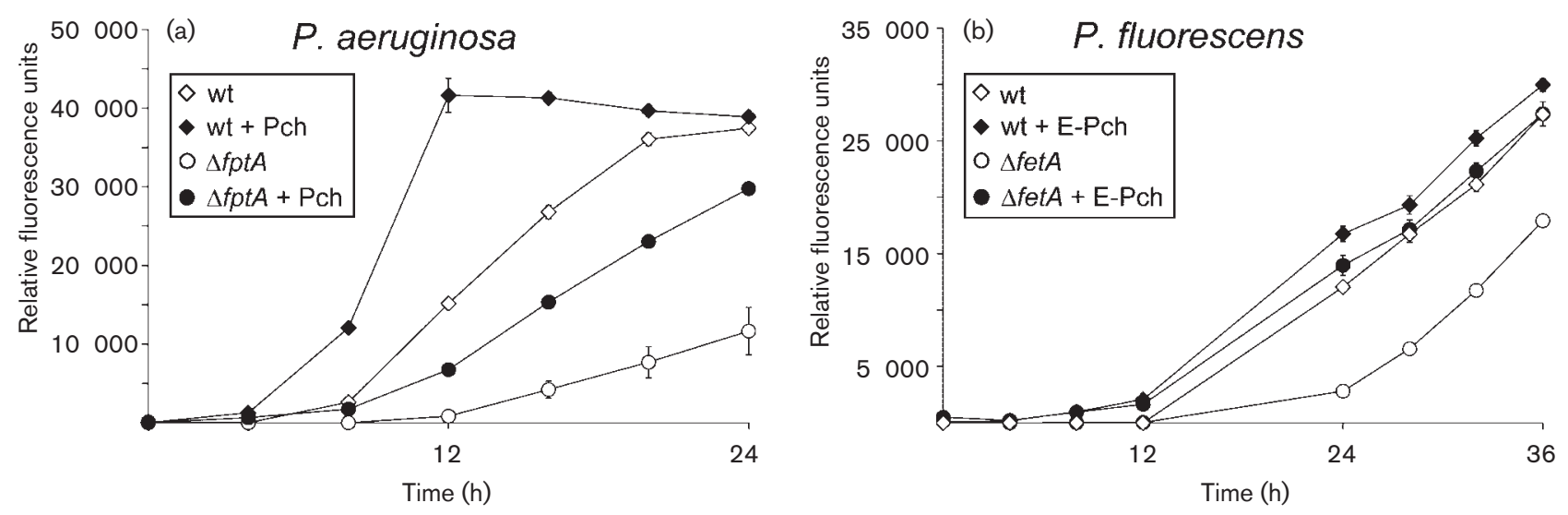

Fig. 4. The pyochelin/enantio-pyochelin receptors FptA/FetA contribute to, but are not essential for, siderophore signalling. (a) The $P$. aeruginosa wild-type (wt) strain PAO1 and its receptor-negative mutant PAO6428 $(\triangle f p t A)$ containing either the reporter

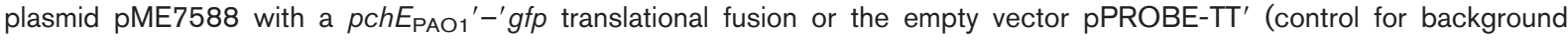
fluorescence) were grown in microtitre wells, and green fluorescence and growth $\left(O D_{600}\right)$ were measured from three parallel experiments. Strains were grown in GGP medium $(\diamond$ for PAO1; $\bigcirc$ for PAO6428) or GGP medium amended with $20 \mu \mathrm{M}$ HPLC-purified pyochelin (Pch) ( for PAO1+Pch; - for PAO6428+Pch). Fluorescence is expressed as relative fluorescence units \pm standard deviation. (b) The same experiment was performed with the $P$. fluorescens wild-type strain (wt) $\mathrm{CHAO}$ and its receptor-negative derivative $\mathrm{CHA} 1086$ ( $\triangle$ fet $A$ ) grown in GGP medium $(\diamond$ for $\mathrm{CHA0} \bigcirc \bigcirc$ for $\mathrm{CHA} 1086)$ or GGP medium with $20 \mu \mathrm{M}$ enantio-pyochelin (E-Pch) ( for CHAO +E-Pch; for CHA1086+E-Pch). Strains carried pME7585 with $p c h D_{\mathrm{CHAO}}{ }^{-' g f p}$ as a reporter or $\mathrm{pPROBE}-\mathrm{TT}^{\prime}$ for measuring background fluorescence. 
(a) 1

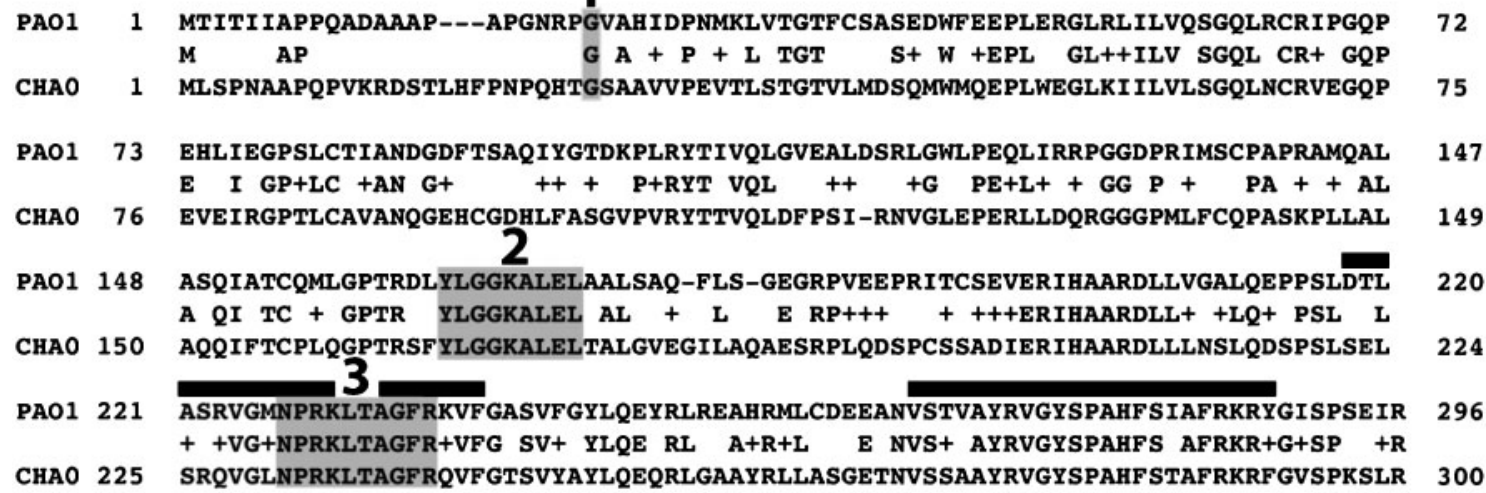

(b)

\begin{tabular}{|c|c|c|c|c|c|}
\hline \multirow{2}{*}{ Plasmid } & \multirow{2}{*}{ Hybrid } & \multicolumn{2}{|c|}{ Amino acids of } & \multicolumn{2}{|c|}{ Complementation of } \\
\hline & & $\operatorname{PchR}_{\mathrm{PAO} 1}$ & $\mathrm{PchR}_{\mathrm{CHAO}}$ & $\begin{array}{r}\text { PAO6402 } \\
\text { (pvd, pchR }\end{array}$ & $\begin{array}{c}\text { CHA1222 } \\
\text { (pvd, pchR) }\end{array}$ \\
\hline pME7547 & & $1-296$ & - & 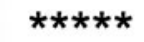 & - \\
\hline pME9254 & & $1-296$ & - & $\star \star \star \star \star *$ & - \\
\hline pME9252 & & - & $1-300$ & - & $\star \star \star \star \star *$ \\
\hline pME9255 & & - & $1-300$ & - & * \\
\hline pME9263 & 1 & $24-296$ & $1-26$ & * & - \\
\hline pME9266 & $1 a$ & $1-23$ & $27-300$ & - & 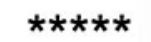 \\
\hline pME9258 & 2 & 169-296 & $1-170$ & - & * \\
\hline pME9269 & $2 a$ & $1-168$ & $171-300$ & $\star \star \star *$ & - \\
\hline pME9260 & 3 & 232-296 & $1-235$ & - & ** \\
\hline pME9261 & $3 a$ & $1-231$ & $236-300$ & $\star \star$ & - \\
\hline
\end{tabular}

Fig. 5. Construction and activity of wild-type and hybrid $p c h R$ genes. (a) The deduced protein sequences of $P$. aeruginosa

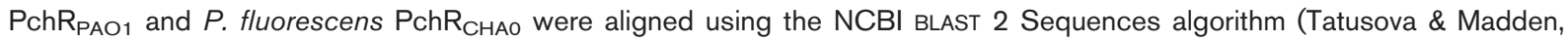
1999). The two helix-turn-helix DNA-binding motifs characteristic of members of the AraC/XylS family (Gallegos et al., 1997) are indicated by black bars above the sequence alignment. The three highlighted regions were selected for the construction of PchR hybrids. (b) The ability of wild-type and hybrid pchR genes to restore pyochelin and enantio-pyochelin production in the pchR mutant strains PAO6402 and CHA1222 was assessed on CAS agar and is indicated by stars, ranging from no stars for no complementation and no siderophore production to five stars for full complementation and wild-type siderophore production. The upstream promoter and leader regions of each gene are represented by a triangle and the $p c h R$ genes are represented by the adjacent rectangle. Sequences of $P$. aeruginosa PAO1 origin are in light grey; sequences from $P$. fluorescens $\mathrm{CHAO}$ are in dark grey. The coordinates of $\mathrm{PchR}_{\mathrm{PAO} 1}$ and $\mathrm{PchR}_{\mathrm{CHAO}}$ amino acids correspond to those shown in (a). For the original CAS agar images on which this figure is based see Supplementary Fig. S1.

regions of identical sequences to create the hybrids. Regions 1 and 2 are predicted to be within the siderophore-binding domain of $\mathrm{PchR}$ while region 3 is at the start of the predicted DNA-binding domain (Fig. 5a). Wild-type and hybrid $p c h R$ genes were integrated in monocopy into the pyoverdine-negative $p c h R$ mutants PAO6402 and CHA1222 and scored for their ability to complement the $p c h R$ mutation and restore production of pyochelin and enantio-pyochelin, respectively. We first tested if swapping the promoter/leader sequences of $p c h R_{\mathrm{PAO} 1}$ and $p c h R_{\mathrm{CHA} 0}$ would affect the ability of the two genes to complement $p c h R$ mutations in $P$. aeruginosa and $P$. fluorescens, respectively. As shown in Fig. 5(b) and in Supplementary Fig. S1, pyochelin production was restored in PAO6402 equally well with pME7547 and pME9254, indicating that the $p c h R_{\mathrm{PAO} 1}$ gene is fully 
functional in $P$. aeruginosa when linked to the promoter and leader sequences of $p c h R_{\mathrm{CHA} O}$. In contrast, enantiopyochelin production in CHA1222 with pME9255 was low compared to pME9252 (Fig. 5b and Supplementary Fig. $\mathrm{S} 1)$. For unknown reasons, combining the $p c h R_{\mathrm{PAO} 1}$ upstream region with the coding sequence of $p c h R_{\mathrm{CHA} 0}$ seems to prevent normal pchR expression in $P$. fluorescens. To circumvent this problem, we constructed all hybrid $p c h R$ genes with the promoter and leader region of $p c h R_{\text {CHA0 }}$.

Hybrids 1 and 1a (Fig. 5b) were constructed by an exchange at the conserved glycine residue located at positions 23 and 26, respectively, in $\mathrm{PchR}_{\mathrm{PAO} 1}$ and PchR $_{\text {CHAO }}$ (Fig. 5a). Hybrid 1 was less effective in complementing PAO6402 than wild-type $p c h R_{\mathrm{PAO} 1}$ and non-functional in CHA1222. Hybrid 1a, in contrast, was almost fully functional in CHA1222 but unable to complement the pchR mutation in PAO6402. Thus, the first $\sim 20$ residues of PchR do not seem to be involved in conferring siderophore specificity to $\mathrm{PchR}$ but are functionally important, at least for $\mathrm{PchR}_{\mathrm{PAO} 1}$.

Hybrids 2 and 2a were constructed by an exchange at the conserved sequence YLGGKALEL (Fig. 5a). Hybrid 2 was non-functional in PAO6402 but restored a minor production of enantio-pyochelin in CHA1222, whereas hybrid 2a was non-functional in CHA1222 but partially active in PAO6402 (Fig. 5b; Supplementary Fig. S1). Hybrids 3 and 3 a were constructed by an exchange at a conserved sequence NPRKLTAGFR within the predicted C-terminal domain of PchR (Fig. 5a) and complementation data resembled those obtained with hybrids 2 and 2a. Hybrid 3 partially complemented the pchR mutation in CHA1222, but was not functional in PAO6402, whereas hybrid 3a was unable to complement CHA1222 but showed partial activity in PAO6402 (Fig. 5b; Supplementary Fig. S1).

Together the results obtained with hybrids 2, 2a, 3 and $3 a$ show that the specificity of $\mathrm{PchR}_{\mathrm{PAO}}$ for pyochelin and

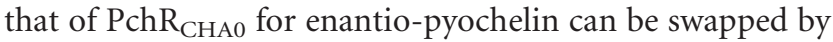
replacing the $\mathrm{N}$-terminal domain of $\mathrm{PchR}$ with that of the other species. More precisely, these data suggest that the residues responsible for siderophore recognition lie between the conserved glycine (G23 in $\mathrm{PchR}_{\mathrm{PAO} 1}$, G26 in $\left.\mathrm{PchR}_{\mathrm{CHA} 0}\right)$ and the conserved sequence YLGGKALEL (164-172 in $\mathrm{PchR}_{\mathrm{PAO} 1}, 166-174$ in $\left.\mathrm{PchR}_{\mathrm{CHAO}}\right)$.

\section{Overexpression of the PchR N-terminal domain reduces siderophore production}

Overexpression of PchR derivatives lacking the C-terminal DNA-binding domain from an IPTG-inducible tac promoter interfered with siderophore production. This was seen in strain PAO6382, where pyochelin production was strongly reduced by overexpressing the first 203 amino acids of $\mathrm{PchR}_{\mathrm{PAO} 1}\left(\mathrm{~N}-\mathrm{PchR}_{\mathrm{PAOl}}\right)$, whereas overexpression of the first 207 amino acids of $\mathrm{PchR}_{\mathrm{CHA}}\left(\mathrm{N}-\mathrm{Pch} \mathrm{CHAO}_{\mathrm{CH}}\right)$ had no effect (Fig. 6). Likewise, overexpression of N-
$\mathrm{PchR}_{\mathrm{CHA} 0}$, but not that of $\mathrm{N}-\mathrm{PchR}_{\mathrm{PAOl}}$, reduced the production of enantio-pyochelin in CHA400 (Fig. 6). These results show that overexpression of the N-terminal part of either PchR protein inhibits the production of the corresponding siderophore, but does not interfere with siderophore production in the other species.

\section{DISCUSSION}

Stereospecificity in pyochelin/enantio-pyochelin signalling was thought to be caused by the reported stereospecificity of the FptA/FetA outer-membrane receptors (Hoegy et al., 2009), which would prevent the heterologous siderophore from entering the cell and activating the PchR regulatory protein if no other siderophore uptake system were present. In this study we have shown, however, that stereospecificity in pyochelin/enantio-pyochelin signalling is additionally conferred by the transcriptional regulator PchR. Through the construction of hybrid P. aeruginosa/ $P$. fluorescens PchR proteins we have demonstrated that the region important for recognition of pyochelin/enantiopyochelin is located in the N-terminal part of PchR. Moreover, overexpressed N-terminal parts of $\mathrm{PchR}_{\mathrm{PAO} 1}$ and $\mathrm{PchR}_{\mathrm{CHAO}}$ specifically inhibited the production of their native siderophores.

It appears that stereospecificity of $\mathrm{PchR}_{\mathrm{CHAO}}$ is less stringent than that of $\mathrm{PchR}_{\mathrm{PAO} 1}$, as the $P$. fluorescens protein was activated not only by enantio-pyochelin but to a minor extent also by pyochelin. In contrast, the activity of the $P$. aeruginosa protein was strictly dependent on pyochelin (Fig. 3; Youard et al., 2007). It is not known,

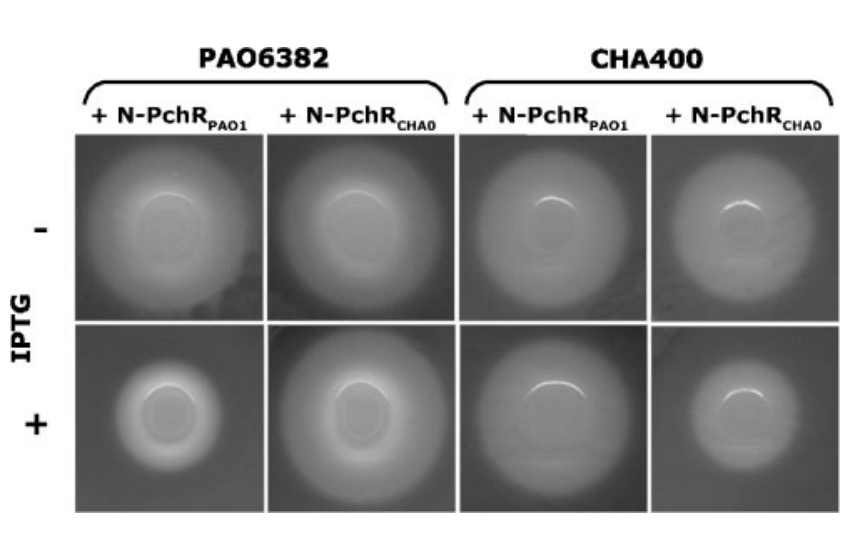

Fig. 6. Inhibition of siderophore production by N-terminal PchR proteins. Pyoverdine-negative strains of $P$. aeruginosa (PAO6382) and $P$. fluorescens (CHA400) containing the N-terminal PchR expression plasmids pME9264 (for overexpression of $\mathrm{N}$ $P_{c h R_{P A O 1}}$ ) and pME9265 (for overexpression of N-PchR $R_{\mathrm{CHAO}}$ ) were grown overnight in GGP medium or GGP medium supplemented with $1 \mathrm{mM}$ IPTG and tested for siderophore production on IPTG-surface-spread or IPTG-free CAS agar plates. Production of pyochelin/enantio-pyochelin can be visualized by the presence of an orange halo surrounding the colony. The experiment was repeated twice with very similar results. 
however, if the expression levels of the two proteins are comparable in the two species, making a strong statement on this issue premature. Siderophore binding studies with purified PchR proteins in vitro will address this point in the future.

As shown in Fig. 3, the PchR proteins of $P$. aeruginosa and $P$. fluorescens were functional as regulators in the heterologous species when their native siderophore was supplied in the growth medium. Given that the $P$. aeruginosa outermembrane receptor FptA is specific for pyochelin and the $P$. fluorescens receptor FetA is specific for enantio-pyochelin (Hoegy et al., 2009), this implies that both siderophores are able to enter cells via another route at concentrations that are sufficient to permit PchR activation. Indeed, previous experiments performed in P. aeruginosa (Michel et al., 2007) and the results shown in Fig. 4 confirm that mutations in the pyochelin and enantio-pyochelin receptor genes $f p t A$ and fetA, respectively, delay, but do not abolish, signalling in $P$. aeruginosa and $P$. fluorescens. By contrast, deletion of the pyochelin and enantio-pyochelin receptors prevents Pseudomonas from utilizing pyochelin and enantio-pyochelin for iron uptake (Michel et al., 2007; Youard et al., 2007). Therefore, it appears that pyochelin/enantio-pyochelin signalling requires a lower extracellular siderophore concentration than does growth promotion by pyochelin/ enantio-pyochelin. Similar findings were reported for the siderophore yersiniabactin in Yersinia pestis, where transcriptional activation of the yersiniabactin biosynthesis genes by the PchR-related AraC-type regulator YbtA required a 500-fold lower siderophore concentration than was required for growth stimulation. Interestingly, however, mutations in the $Y$. pestis siderophore uptake genes did not reduce or delay transcriptional activation of the yersiniabactin genes, suggesting that receptor bypass of the siderophore in $Y$. pestis is even more efficient than in Pseudomonas and allows full activation of the YbtA regulator (Perry et al., 2003).

Swapping the first $\sim 170$ residues of $\mathrm{PchR}$ changed the siderophore specificity of $\mathrm{PchR}_{\mathrm{PAO}}$ and $\mathrm{PchR}_{\mathrm{CHAO}}$ (Fig. 5, hybrids 2 and $2 \mathrm{a}$ ) while the $\sim 20 \mathrm{~N}$-terminal amino acids were not important (Fig. 5, hybrids 1 and 1a). These results indicate that key residues forming the siderophore-binding pocket of PchR lie in the region between residues $\sim 20$ and $\sim 170$. Except for hybrid 1a, none of the hybrids completely restored pyochelin or enantio-pyochelin production (Fig. 5b and Supplementary Fig. S1). This may not be too surprising, as the assay system used (siderophore production on CAS agar) does not directly measure siderophore binding to wild-type and hybrid PchR proteins but integrates the whole response on siderophore biosynthesis and export, meaning that minor changes in PchR activity or stability can have a large impact.

Overexpression of the N-terminal domains of $\mathrm{PchR}_{\mathrm{PAO} 1}$ and $\mathrm{PchR}_{\mathrm{CHA} 0}$ inhibited the function of their corresponding full-length proteins. At this stage it is uncertain whether inhibition is caused by binding and sequestering of the respective siderophore, by physical interaction with the corresponding full-length PchR protein, or by a combination of both mechanisms. An interaction of N-PchR with PchR would support the idea that PchR functions as a dimer or multimer whereas siderophore titration seems less likely because siderophore production was still quite high, as evidenced by the orange halo produced on CAS agar (Fig. 6).

In conclusion, we have shown that PchR plays a crucial role in the stereospecificity of pyochelin and enantio-pyochelin signalling in Pseudomonas spp. and that the N-terminal part of PchR is responsible for siderophore recognition.

\section{ACKNOWLEDGEMENTS}

We thank Laurent Michel for providing PAO6402, Gaëtan Cherbuin for constructing pME9258, and Dieter Haas for helpful suggestions and for carefully reading the manuscript. This work was supported by the Swiss National Science Foundation for Scientific Research (project 3100A0-113955/1)

\section{REFERENCES}

Ankenbauer, R. G. \& Quan, H. N. (1994). FptA, the Fe(III)-pyochelin receptor of Pseudomonas aeruginosa: a phenolate siderophore receptor homologous to hydroxamate siderophore receptors. J Bacteriol 176, 307-319.

Ankenbauer, R. G., Toyokuni, T., Staley, A., Rinehart, K. L., Jr \& Cox, C. D. (1988). Synthesis and biological activity of pyochelin, a siderophore of Pseudomonas aeruginosa. J Bacteriol 170, 5344-5351.

Bao, Y., Lies, D. P., Fu, H. \& Roberts, G. P. (1991). An improved Tn7based system for the single-copy insertion of cloned genes into chromosomes of gram-negative bacteria. Gene 109, 167-168.

Carmi, R., Carmeli, S., Levy, E. \& Gough, F. J. (1994). (+)-(S)Dihydroaeruginoic acid, an inhibitor of Septoria tritici and other phytopathogenic fungi and bacteria, produced by Pseudomonas fluorescens. J Nat Prod 57, 1200-1205.

Castignetti, D. (1997). Probing of Pseudomonas aeruginosa, Pseudomonas aureofaciens, Burkholderia (Pseudomonas) cepacia, Pseudomonas fluorescens, and Pseudomonas putida with the ferripyochelin receptor A gene and the synthesis of pyochelin in Pseudomonas aureofaciens, Pseudomonas fluorescens, and Pseudomonas putida. Curr Microbiol 34, 250-257.

Chipperfield, J. R. \& Ratledge, C. (2000). Salicylic acid is not a bacterial siderophore: a theoretical study. Biometals 13, 165-168.

Cox, C. D. \& Graham, R. (1979). Isolation of an iron-binding compound from Pseudomonas aeruginosa. J Bacteriol 137, 357-364.

Cox, C. D., Rinehart, K. L., Jr, Moore, M. L. \& Cook, J. C., Jr (1981). Pyochelin: novel structure of an iron-chelating growth promoter for Pseudomonas aeruginosa. Proc Natl Acad Sci U S A 78, 4256-4260.

Darling, P., Chan, M., Cox, A. D. \& Sokol, P. A. (1998). Siderophore production by cystic fibrosis isolates of Burkholderia cepacia. Infect Immun 66, 874-877.

Farinha, M. A. \& Kropinski, A. M. (1990). High efficiency electroporation of Pseudomonas aeruginosa using frozen cell suspensions. FEMS Microbiol Lett 58, 221-225.

Gallegos, M.-T., Schleif, R., Bairoch, A., Hofmann, K. \& Ramos, J. L. (1997). AraC/XylS family of transcriptional regulators. Microbiol Mol Biol Rev 61, 393-410. 
Heeb, S., Blumer, C. \& Haas, D. (2002). Regulatory RNA as mediator in GacA/RsmA-dependent global control of exoproduct formation in Pseudomonas fluorescens CHA0. J Bacteriol 184, 1046-1056.

Heinrichs, D. E. \& Poole, K. (1993). Cloning and sequence analysis of a gene ( $p c h R)$ encoding an AraC family activator of pyochelin and ferripyochelin receptor synthesis in Pseudomonas aeruginosa. J Bacteriol 175, 5882-5889.

Hoegy, F., Lee, X., Noel, S., Rognan, D., Mislin, G. L., Reimmann, C. \& Schalk, I. J. (2009). Stereospecificity of the siderophore pyochelin outer membrane transporters in fluorescent pseudomonads. J Biol Chem 284, 14949-14957.

Keel, C., Voisard, C., Berling, C., Kahr, G. \& Défago, G. (1989). Iron sufficiency, a prerequisite for the suppression of tobacco black root rot by Pseudomonas fluorescens strain CHA0 under gnotobiotic conditions. Phytopathology 79, 584-589.

Laville, J., Blumer, C., Von Schroetter, C., Gaia, V., Defago, G., Keel, C. \& Haas, D. (1998). Characterization of the $h c n A B C$ gene cluster encoding hydrogen cyanide synthase and anaerobic regulation by ANR in the strictly aerobic biocontrol agent Pseudomonas fluorescens CHA0. J Bacteriol 180, 3187-3196.

Michel, L., González, N., Jagdeep, S., Nguyen-Ngoc, T. \& Reimmann, C. (2005). PchR-box recognition by the AraC-type regulator PchR of Pseudomonas aeruginosa requires the siderophore pyochelin as an effector. Mol Microbiol 58, 495-509.

Michel, L., Bachelard, A. \& Reimmann, C. (2007). Ferripyochelin uptake genes are involved in pyochelin-mediated signalling in Pseudomonas aeruginosa. Microbiology 153, 1508-1518.

Miller, W. G., Leveau, J. H. \& Lindow, S. E. (2000). Improved $g f p$ and inaZ broad-host-range promoter-probe vectors. Mol Plant Microbe Interact 13, 1243-1250.

Ó Cuív, P., Clarke, P., Lynch, D. \& O’Connell, M. (2004). Identification of $r h t X$ and $f p t X$, novel genes encoding proteins that show homology and function in the utilization of the siderophores rhizobactin 1021 by Sinorhizobium meliloti and pyochelin by Pseudomonas aeruginosa, respectively. J Bacteriol 186, 2996-3005.

Perry, R. D., Abney, J., Mier, I., Jr, Lee, Y., Bearden, S. W. \& Fetherston, J. D. (2003). Regulation of the Yersinia pestis Yfe and Ybt iron transport systems. Adv Exp Med Biol 529, 275-283.

Reimmann, C., Serino, L., Beyeler, M. \& Haas, D. (1998). Dihydroaeruginoic acid synthetase and pyochelin synthetase, products of the $p c h E F$ genes, are induced by extracellular pyochelin in Pseudomonas aeruginosa. Microbiology 144, 3135-3148.

Reimmann, C., Patel, H. M., Serino, L., Barone, M., Walsh, C. T. \& Haas, D. (2001). Essential PchG-dependent reduction in pyochelin biosynthesis of Pseudomonas aeruginosa. J Bacteriol 183, 813-820.

Rinehart, K. L., Staley, A. L., Wilson, S. R., Ankenbauer, R. G. \& Cox, C. D. (1995). Stereochemical assignment of the pyochelins. J Org Chem 60, 2786-2791.

Ruiz, R., Marqués, S. \& Ramos, J. L. (2003). Leucines 193 and 194 at the N-terminal domain of the XylS protein, the positive transcriptional regulator of the TOL meta-cleavage pathway, are involved in dimerization. J Bacteriol 185, 3036-3041.

Sambrook, J. \& Russell, D. W. (2001). Molecular Cloning: a Laboratory Manual, 3rd edn. Cold Spring Harbor, NY: Cold Spring Harbor Laboratory.

Schlegel, K., Taraz, K. \& Budzikiewicz, H. (2004). The stereoisomers of pyochelin, a siderophore of Pseudomonas aeruginosa. Biometals 17, 409-414.
Schlegel, K., Lex, J., Taraz, K. \& Budzikiewicz, H. (2006). The X-ray structure of the pyochelin $\mathrm{Fe}^{3+}$ complex. $Z$ Naturforsch $[C] 61,263-$ 266.

Schleif, R. (2003). AraC protein: a love-hate relationship. Bioessays 25, 274-282.

Schnider, U., Keel, C., Blumer, C., Troxler, J., Defago, G. \& Haas, D. (1995). Amplification of the housekeeping sigma factor in Pseudomonas fluorescens CHA0 enhances antibiotic production and improves biocontrol abilities. J Bacteriol 177, 5387-5392.

Schwyn, B. \& Neilands, J. B. (1987). Universal chemical assay for the detection and determination of siderophores. Anal Biochem 160, 4756.

Serino, L., Reimmann, C., Baur, H., Beyeler, M., Visca, P. \& Haas, D. (1995). Structural genes for salicylate biosynthesis from chorismate in Pseudomonas aeruginosa. Mol Gen Genet 249, 217-228.

Serino, L., Reimmann, C., Visca, P., Beyeler, M., della Chiesa, V. \& Haas, D. (1997). Biosynthesis of pyochelin and dihydroaeruginoic acid requires the iron-regulated pchDCBA operon in Pseudomonas aeruginosa. J Bacteriol 179, 248-257.

Sokol, P. A. (1986). Production and utilization of pyochelin by clinical isolates of Pseudomonas cepacia. J Clin Microbiol 23, 560-562.

Stanisich, V. A. \& Holloway, B. W. (1972). A mutant sex factor of Pseudomonas aeruginosa. Genet Res 19, 91-108.

Tatusova, T. A. \& Madden, T. L. (1999). BLAST 2 Sequences, a new tool for comparing protein and nucleotide sequences. FEMS Microbiol Lett 174, 247-250.

Tseng, C. F., Burger, A., Mislin, G. L., Schalk, I. J., Yu, S. S., Chan, S. I. \& Abdallah, M. A. (2006). Bacterial siderophores: the solution stoichiometry and coordination of the $\mathrm{Fe}$ (III) complexes of pyochelin and related compounds. J Biol Inorg Chem 11, 419-432.

Visca, P., Imperi, F. \& Lamont, I. L. (2007). Pyoverdine siderophores: from biogenesis to biosignificance. Trends Microbiol 15, 22-30.

Voisard, C., Rella, M. \& Haas, D. (1988). Conjugative transfer of plasmid RP1 to soil isolates of Pseudomonas fluorescens is facilitated by certain large RP1 deletions. FEMS Microbiol Lett 55, 9-13.

Voisard, C., Bull, C. T., Keel, C., Laville, J., Maurhofer, M., Schnider, U., Défago, G. \& Haas, D. (1994). Biocontrol of root diseases by Pseudomonas fluorescens CHA0: current concepts and experimental approaches. In Molecular Ecology of Rhizosphere Microorganisms, pp. 67-89. Edited by F. O'Gara, D. N. Dowling \& B. Boesten. Weinheim, Germany: VCH Publishers.

Ye, R. W., Haas, D., Ka, J. O., Krishnapillai, V., Zimmermann, A., Baird, C. \& Tiedje, J. M. (1995). Anaerobic activation of the entire denitrification pathway in Pseudomonas aeruginosa requires Anr, an analog of Fnr. J Bacteriol 177, 3606-3609.

Youard, Z. A., Mislin, G. L., Majcherczyk, P. A., Schalk, I. J. \& Reimmann, C. (2007). Pseudomonas fluorescens CHA0 produces enantio-pyochelin, the optical antipode of the Pseudomonas aeruginosa siderophore pyochelin. J Biol Chem 282, 35546-35553.

Zuber, S., Carruthers, F., Keel, C., Mattard, A., Blumer, C., Pessi, G., Gigot-Bonnefoy, C., Schnider-Keel, U., Heeb, S. \& other authors (2003). GacS sensor domains pertinent to the regulation of exoproduct formation and to the biocontrol potential of Pseudomonas fluorescens CHA0. Mol Plant Microbe Interact 16, 634644.

Edited by: W. Bitter 